\title{
Non-Adiabatic Quantities in $\delta$ Scuti and $\gamma$ Dor Stars: a Comparison between Observations and Theoretical Non-Adiabatic Models
}

\author{
R. Garrido ${ }^{1}$, A. Moya, A. Claret \\ Instituto de Astrofisica de Andalucia, CSIC, Apdo 3004, 18080, \\ Granada, Spain
}

\begin{abstract}
Phase lag, $\Psi^{T}$ and $R$, a parameter measuring the departure from adiabaticity, can be derived directly from multicolor photometry. The same quantities can also be calculated from theoretical non-adiabatic pulsation models. We present here some preliminary theoretical results which indicate that these quantities depend on the mixing length parameter used to treat convection in the standard ML theory. The comparison favours models with $\alpha$ values between 1 and $1.5 \mathrm{Hp}$. Non-adiabatic models also predict the observed phase lags for the newly discovered $\gamma$ Dor g-mode pulsating stars.
\end{abstract}

\section{Introduction}

The linear aproximation to radial and non-radial flux variations of a pulsating star was first derived by Dziembowski (1977) and then reformulated by Balona and Stobie (1979a,b) and by Watson (1988) in the form which we are using now. Several attempts to fit real observations, FG Vir in Breger et al. (1999), BI CMi in Breger et al. (2001a), 44 Tau in Breger et al. (2001b), V1162 Ori in Arentoft et al. (2001) and several other stars in Garrido (2000) have shown that the method can be useful at least for low rotational velocities. For one star $-20 \mathrm{CVn}$ - spectroscopic observations by Chadid et al. (2001) confirm results previously found from multicolor photometry - presented by Rodriguez et al. (1994) - in the sense that this star is a radially pulsating object.

The main difficulty of the method lies in the high precision needed for the individual phases of each photometric band, usually better than 1 degree. Garrido et al. (1990) also showed that for low $\ell$ values the wavelength dependence of the limb darkening integrals appearing in the linearised formula is very weak, allowing combinations of several colors - at least three distributed in a range as wide as possible - to give consistent values for the couple $\left(\Psi^{T}, R\right)$. Results for $\delta$ Scuti and $\gamma$ Dor stars were taken from Garrido (2000) and have to be compared with the non-adiabatic theoretical results presented in the following section.

1e-mail: garrido@iaa.es 


\section{Non-adiabatic non-radial theoretical models}

The equilibrium models we are using here have been extensively used for other purposes. They have mainly been used to predict properties of double-lined eclipsing binaries and to compare theoretical mass distributions with those deduced from observed apsidal motion rates (Claret, 1995, 1997). Adiabatic and non-adiabatic codes have been developed following Unno et al. (1989). The inclusion of non-adiabatic calculations allows us to derive values for the couple $\left(\Psi^{T}, R\right)$. Boundary conditions are those used in Unno et al. but with a different normalization, we use $y_{2} \equiv\left(p^{\prime} / \rho+\phi^{\prime}\right) / g r=1$ at $r=0$. The effect of different boundary conditions is now under study. Rotation and interaction convection-pulsation are completely ignored in this study. Stars for which we can derive reliable $\left(\Psi^{T}, R\right)$ values are the so-called High Amplitude Delta Scuti Stars (HADS) since we need a very high precision in order to derive phase differences among different colors.

\section{Comparison with observations}

Fig. 1 shows a general comparison of the data, as deduced from Strömgren 4color photometry, with theoretical predictions. Note the fair agreement with observations in the wide range described by the $\delta$ Scuti stars on one side and the $\gamma$ Dor on the other. Observations and theoretical predictions point towards $\Psi^{T}=0^{0}$ for the newly discovered $\gamma$ Dor stars and, as it was known, an angle around $102^{0}$ for $\delta$ Scuti stars. It is also to be noted here that predictions give $R$ values close to those observed. The non-adiabatic effects are seen mainly in the eigenfunction describing the temperature perturbations and originate in the regions where opacity bumps exist. The reason why the phase of the radial perturbations follows the temperature perturbations for the g-modes is the proximity of a node. In general, the phase angle $\Psi^{T}$ changes from $-\pi$ to $\pi$ when one goes from a high order g-mode to a high order p-mode and the physical reason is the choice of the external boundary condition. The form in which the eigenvalue appears in the formula implies a jump in the relationship between temperature and gravity relative perturbations, dominating the behaviour of the non-adiabatic couple $\left(\Psi^{T}, R\right)$. The physical reality of this boundary condition can be observationally tested by measuring these non-adiabatic quantities from multicolor observations. The effect of different mixing length $\alpha$ parameters on $\left(\Psi^{T}, R\right)$ becomes more important as the convection zone grows larger. The effect is dramatic for a $1.6 M_{\odot}$ model, typical of a $\gamma$ Dor star. Nevertheless even for hotter and non-convective stars, such as the $\beta$ Cephei stars, the effect on the color phases could be important for modes which are not centered around the fundamental radial, for which the predicted phase differences are very small.

Future asteroseismology space missions, such as COROT and MONS where color is implemented, will supply - because of the expected high precision for phase differences in different colors - an important database to test real star atmospheres through the study of their non-adiabatic properties. 


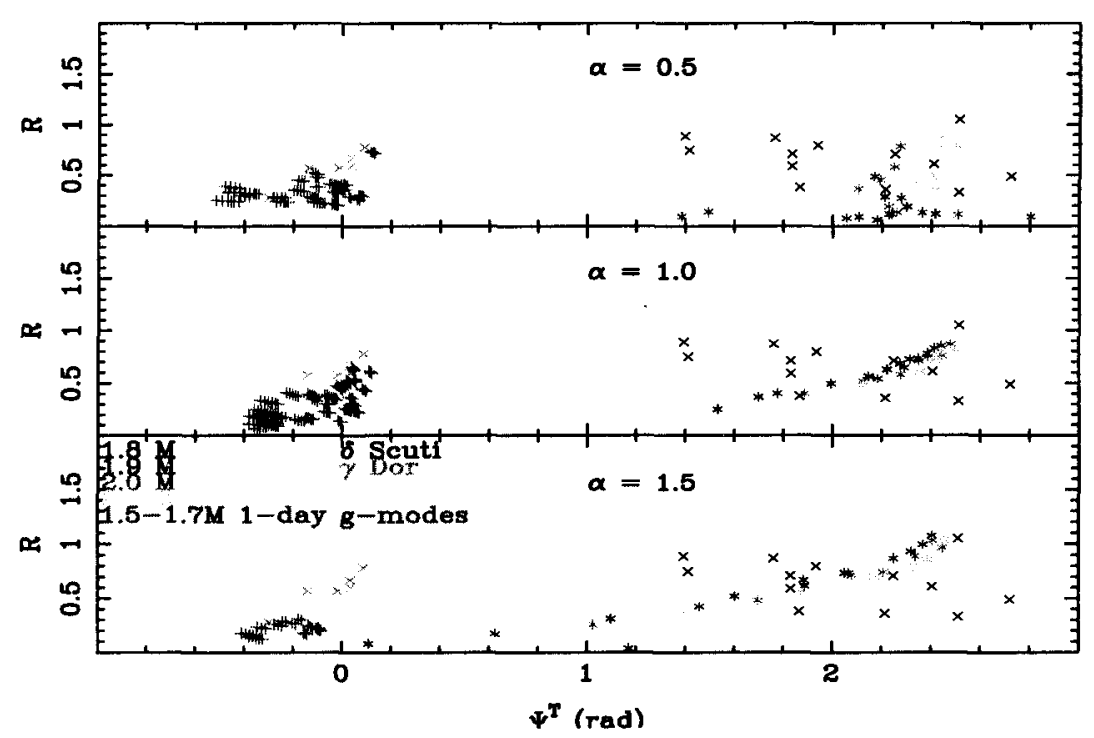

Figure 1. General comparison of the data, as deduced from Strömgren 4-color photometry, with theoretical predictions.

\section{References}

Arentoft, T., Sterken, C., Handler, G., et al. 2001, A\&A, 374, 1056

Balona, L.A. \& Stobie, R.S. 1979a, MNRAS, 189, 627

Balona, L.A. \& Stobie, R.S. 1979b, MNRAS, 189, 649

Breger, M., et al. 2001a, A\&A, in preparation

Breger, M., Handler, G., Garrido, R., et al. 2001b, MNRAS, in press

Breger, M., Pamyatnykh, A.A., Pikall, H. \& Garrido, R. 1999, A\&A, 341, 151

Chadid, M., De Ridder, J., Aerts, C., \& Mathias, P. 2001, A\&A, 375, 113

Claret, A. 1995, A\&AS, 109, 441

Claret, A. 1997, A\&AS, 327, 11

Dziembowski, W. 1977, Acta Astron., 27, 203

Garrido, R. 2000, in ASP Conf. Ser., Vol. 210, Delta Scuti and Related Stars, eds. M. Breger \& M. Montgomery, (San Francisco ASP), 67

Garrido, R., Garcia-Lobo, E., \& Rodriguez, E. 1990, A\&A, 234, 262

Rodriguez, E., Lopez de Coca, P., Rolland, A., Garrido, R. \& Costa, V. 1994, A\&AS, 106, 21

Unno, W., Osaki, Y., \&o, H., Saio, H. \& Shibahashi, H., Nonradial oscillations of stars, (Univ. Tokyo Press)

Watson, R.D. 1988, Ap\&SS, 140, 255 